\title{
Argument Estimates of Multivalent Functions Involving a Certain Fractional Derivative Operator
}

\section{Jae Ho Choi}

Department of Mathematics Education, Daegu National University of Education, Daegu, South Korea Email: choijh@dnue.ac.kr

Received 18 January 2015; accepted 9 February 2015; published 13 February 2015

Copyright (C) 2015 by author and Scientific Research Publishing Inc.

This work is licensed under the Creative Commons Attribution International License (CC BY).

http://creativecommons.org/licenses/by/4.0/

\section{(c) (i) Open Access}

\section{Abstract}

The object of the present paper is to investigate various argument results of analytic and multivalent functions which are defined by using a certain fractional derivative operator. Some interesting applications are also considered.

\section{Keywords}

\section{Multivalent Analytic Functions, Argument, Integral Operator, Fractional Derivative Operator}

\section{Introduction}

Let $\mathcal{A}(p)$ denote the class of functions $f(z)$ of the form

$$
f(z)=z^{p}+\sum_{k=1}^{\infty} a_{p+k} z^{p+k}, \quad(p \in \mathbb{N}:=\{1,2,3, \cdots\}),
$$

which are analytic in the open unit disk $\mathbb{U}=\{z: z \in \mathbb{C}$ and $|z|<1\}$. Also let $\mathcal{A}(1)=\mathcal{A}$ denote the class of all analytic functions $p(z)$ with $p(0)=1$ which are defined on $\mathbb{U}$.

Let $a, b$ and $c$ be complex numbers with $c \neq 0,-1,-2, \cdots$. Then the Gaussian hypergeometric function ${ }_{2} F_{1}(a, b ; c ; z)$ is defined by

$$
{ }_{2} F_{1}(a, b ; c ; z)=\sum_{k=0}^{\infty} \frac{(a)_{k}(b)_{k}}{(c)_{k}} \frac{z^{k}}{k !},
$$

where $(\eta)_{k}$ is the Pochhammer symbol defined, in terms of the Gamma function, by 


$$
(\eta)_{k}=\frac{\Gamma(\eta+k)}{\Gamma(\eta)}= \begin{cases}1, & (k=0) ; \\ \eta(\eta+1) \cdots(\eta+k-1), & (k \in \mathbb{N}) .\end{cases}
$$

The hypergeometric function ${ }_{2} F_{1}(a, b ; c ; z)$ is analytic in $\mathbb{U}$ and if $a$ or $b$ is a negative integer, then it reduces to a polynomial.

There are a number of definitions for fractional calculus operators in the literature (cf., e.g., [1] and [2]). We use here the Saigo type fractional derivative operator defined as follows ([3]; see also [4]):

Definition 1. Let $0 \leq \lambda<1$ and $\mu, v \in \mathbb{R}$. Then the generalized fractional derivative operator $\mathcal{J}_{0, z}^{\lambda, \mu, v}$ of a function $f(z)$ is defined by

$$
\mathcal{J}_{0, z}^{\lambda, \mu, v} f(z)=\frac{\mathrm{d}}{\mathrm{d} z}\left(\frac{z^{\lambda-\mu}}{\Gamma(1-\lambda)} \int_{0}^{z}(z-\zeta)^{-\lambda}{ }_{2} F_{1}\left(\mu-\lambda, 1-v ; 1-\lambda ; 1-\frac{\zeta}{z}\right) f(\zeta) \mathrm{d} \zeta\right)
$$

The function $f(z)$ is an analytic function in a simply-connected region of the z-plane containing the origin, with the order

$$
f(z)=O\left(|z|^{\epsilon}\right), \quad(z \rightarrow 0)
$$

for $\epsilon>\max \{0, \mu-v\}-1$, and the multiplicity of $(z-\zeta)^{-\lambda}$ is removed by requiring that $\log (z-\zeta)$ to be real when $z-\zeta>0$.

Definition 2. Under the hypotheses of Definition 1, the fractional derivative operator $\mathcal{J}_{0, z}^{\lambda+m, \mu+m, v+m}$ of a function $f(z)$ is defined by

$$
\mathcal{J}_{0, z}^{\lambda+m, \mu+m, v+m} f(z)=\frac{\mathrm{d}^{m}}{\mathrm{~d} z^{m}} \mathcal{J}_{0, z}^{\lambda, \mu, v} f(z), \quad\left(z \in \mathbb{U} ; m \in \mathbb{N}_{0}:=\mathbb{N} \cup\{0\}\right)
$$

With the aid of the above definitions, we define a modification of the fractional derivative operator $\Delta_{z, p}^{\lambda, \mu, v}$ by

$$
\Delta_{z, p}^{\lambda, \mu, v} f(z)=\frac{\Gamma(p+1-\mu) \Gamma(p+1-\lambda+v)}{\Gamma(p+1) \Gamma(p+1-\mu+v)} z^{\mu} \mathcal{J}_{0, z}^{\lambda, \mu, v} f(z)
$$

for $f(z) \in \mathcal{A}(p)$ and $\mu-v-p<1$. Then it is observed that $\Delta_{z, p}^{\lambda, \mu, v}$ also maps $\mathcal{A}(p)$ onto itself as follows:

$$
\begin{aligned}
& \Delta_{z, p}^{\lambda, \mu, v} f(z)=z^{p}+\sum_{k=1}^{\infty} \frac{(p+1)_{k}(p+1-\mu+v)_{k}}{(p+1-\mu)_{k}(p+1-\lambda+v)_{k}} a_{k+p} z^{k+p}, \\
& (z \in \mathbb{U} ; 0 \leq \lambda<1 ; \mu-v-p<1 ; f \in \mathcal{A}(p)) .
\end{aligned}
$$

It is easily verified from (1.6) that

$$
z\left(\Delta_{z, p}^{\lambda, \mu, v} f(z)\right)^{\prime}=(p-\mu) \Delta_{z, p}^{\lambda+1, \mu+1, v+1} f(z)+\mu \Delta_{z, p}^{\lambda, \mu, v} f(z) .
$$

Note that $\Delta_{z, p}^{0,0, v} f=f, \quad \Delta_{z, p}^{1,1, v} f=z f^{\prime} / p$ and $\Delta_{z, p}^{\lambda, \lambda, v} f=\Omega_{z}^{(\lambda, p)} f$, where $\Omega_{z}^{(\lambda, p)}$ is the fractional derivative operator defined by Srivastava and Aouf [5].

In this manuscript, we drive interesting argument results of multivalent functions defined by fractional derivative operator $\Delta_{z, p}^{\lambda, \mu, \nu}$.

\section{Main Results}

In order to establish our results, we require the following lemma due to Lashin [6].

Lemma 1 [6]. Let $h(z)$ be analytic in $\mathbb{U}$, with $h(0)=1$ and $h(z) \neq 0 \quad(z \in \mathbb{U})$. Further suppose that $\alpha, \beta \in \mathbb{R}^{+}=(0, \infty)$ and

$$
\left|\arg \left(h(z)+\beta z h^{\prime}(z)\right)\right|<\frac{\pi}{2}\left(\alpha+\frac{2}{\pi} \arctan (\beta \alpha)\right) \quad(\alpha>0 ; \beta>0)
$$

then 


$$
|\operatorname{argh}(z)|<\frac{\pi}{2} \alpha, \quad(z \in \mathbb{U})
$$

We begin by proving the following result.

Theorem 1. Let $\lambda \geq 0, \mu<\min \{v+p+1, p\}$ and $\alpha, \gamma, \delta \in \mathbb{R}^{+}$, and let $g(z) \in \mathcal{A}(p)$. Suppose that $f(z) \in \mathcal{A}(p)$ satisfies the condition

$$
\begin{aligned}
& \left|\arg \left(\left\{\frac{\Delta_{z, p}^{\lambda, \mu, v} f(z)}{\Delta_{z, p}^{\lambda, \mu, v} g(z)}\right\}^{\gamma}\left\{1+\delta\left(\frac{\Delta_{z, p}^{\lambda+1, \mu+1, v+1} f(z)}{\Delta_{z, p}^{\lambda, \mu, v} f(z)}-\frac{\Delta_{z, p}^{\lambda+1, \mu+1, v+1} g(z)}{\Delta_{z, p}^{\lambda, \mu, v} g(z)}\right)\right\}\right)\right| \\
& <\frac{\pi}{2}\left(\alpha+\frac{2}{\pi} \arctan \left[\frac{\delta}{\gamma(p-\mu)} \alpha\right]\right),
\end{aligned}
$$

then

$$
\left|\arg \left\{\frac{\Delta_{z, p}^{\lambda, \mu, v} f(z)}{\Delta_{z, p}^{\lambda, \mu, v} g(z)}\right\}^{\gamma}\right|<\frac{\pi}{2} \alpha, \quad(z \in \mathbb{U}) .
$$

Proof. If we define the function $h(z)$ by

$$
h(z)=\left\{\frac{\Delta_{z, p}^{\lambda, \mu, v} f(z)}{\Delta_{z, p}^{\lambda, \mu, v} g(z)}\right\}^{\gamma}, \quad(\gamma \neq 0),
$$

then $h(z)=1+c_{1} z+c_{2} z^{2}+\cdots$ is analytic in $\mathbb{U}$, with $h(0)=1$ and $h^{\prime}(0) \neq 0$. Making use of the logarithmic differentiation on both sides of (2.5), we have

$$
\frac{1}{\gamma} \frac{z h^{\prime}(z)}{h(z)}=\frac{z\left(\Delta_{z, p}^{\lambda, \mu, v} f(z)\right)^{\prime}}{\Delta_{z, p}^{\lambda, \mu, v} f(z)}-\frac{z\left(\Delta_{z, p}^{\lambda, \mu, v} g(z)\right)^{\prime}}{\Delta_{z, p}^{\lambda, \mu, v} g(z)} .
$$

By applying the identity (1.7) in (2.6), we observe that

$$
h(z)+\frac{\delta}{\gamma(p-\mu)} z h^{\prime}(z)=\left\{\frac{\Delta_{z, p}^{\lambda, \mu, v} f(z)}{\Delta_{z, p}^{\lambda, \mu, v} g(z)}\right\}^{\gamma}\left\{1+\delta\left(\frac{\Delta_{z, p}^{\lambda+1, \mu+1, v+1} f(z)}{\Delta_{z, p}^{\lambda, \mu, v} f(z)}-\frac{\Delta_{z, p}^{\lambda+1, \mu+1, v+1} g(z)}{\Delta_{z, p}^{\lambda, \mu, v} g(z)}\right)\right\} .
$$

Hence, by using Lemma 1, we conclude that

$$
|\operatorname{argh}(z)|<\frac{\pi}{2} \alpha, \quad(z \in \mathbb{U}),
$$

which completes the proof of Theorem 1 .

Remark 1. Putting $\lambda=\mu=0, \delta=p=1$ and $g(z)=z$ in Theorem 1, we obtain the result due to Lashin ([6], Theorem 2.2).

Taking $\gamma=1$ and $g(z)=z^{p}$ in Theorem 1, we have the following corollary.

Corollary 1. Let $\lambda \geq 0, \mu<\min \{v+p+1, p\}$ and $\alpha, \delta \in \mathbb{R}^{+}$. Suppose that $f(z) \in \mathcal{A}(p)$ satisfies the condition

$$
\left|\arg \left\{(1-\delta) \frac{\Delta_{z, p}^{\lambda, \mu, v} f(z)}{z^{p}}+\delta \frac{\Delta_{z, p}^{\lambda+1, \mu+1, v+1} f(z)}{z^{p}}\right\}\right|<\frac{\pi}{2}\left(\alpha+\frac{2}{\pi} \arctan \left[\frac{\delta \alpha}{p-\mu}\right]\right),
$$

then

$$
\left|\arg \left(\frac{\Delta_{z, p}^{\lambda, \mu, v} f(z)}{z^{p}}\right)\right|<\frac{\pi}{2} \alpha, \quad(z \in \mathbb{U}) .
$$

Theorem 2. Let $\lambda \geq 0, \mu<\min \{v+p+1, p\}, \quad 0<\delta \leq 1$ and $\alpha, \delta \in \mathbb{R}^{+}$. Suppose that $f(z) \in \mathcal{A}(p)$ sa- 
tisfies the condition

$$
\left|\arg \left(\frac{\Delta_{z, p}^{\lambda, \mu, v} f(z)}{z^{p}}\right)\right|<\frac{\pi}{2}\left(\alpha+\frac{2}{\pi} \arctan \left[\frac{\delta}{\gamma(p-\mu)} \alpha\right]\right), \quad(z \in \mathbb{U}) .
$$

then

$$
\left|\arg \left(\frac{\gamma(p-\mu)}{\delta} z^{-\frac{\gamma(p-\mu)}{\delta}} \int_{0}^{z} t^{\frac{\gamma(p-\mu)-\delta(p+1)}{\delta}} \Delta_{z, p}^{\lambda, \mu, v} f(t) \mathrm{d} t\right)\right|<\frac{\pi}{2} \alpha .
$$

Proof. If we set

$$
h(z)=\frac{\gamma(p-\mu)}{\delta} z^{-\frac{\gamma(p-\mu)}{\delta}} \int_{0}^{z} t \frac{\gamma(p-\mu)-\delta(p+1)}{\delta} \Delta_{z, p}^{\lambda, \mu, v} f(t) \mathrm{d} t,
$$

then $h(z)=1+c_{1} z+c_{2} z^{2}+\cdots$ is analytic in $\mathbb{U}$, with $h(0)=1$ and $h^{\prime}(0) \neq 0$. By using the logarithmic differentiation on both sides of (2.9), we obtain

$$
h(z)+\frac{\delta}{\gamma(p-\mu)} z h^{\prime}(z)=\frac{\Delta_{z, p}^{\lambda, \mu, v} f(z)}{z^{p}} .
$$

Thus, in view of Lemma 1, we have

$$
|\operatorname{argh}(z)|<\frac{\pi}{2} \alpha, \quad(z \in \mathbb{U}),
$$

which evidently proves Theorem 2 .

Remark 2. Setting $\lambda=\mu=0$ and $\gamma=\delta=p=1$ in Theorem 2, we get the result obtained by Goyal and Goswami ([7], Corollary 3.6).

Putting $\lambda=\mu=\gamma=\delta=1$ in Theorem 2, we obtain the following result.

Corollary 2. Let $\alpha \in \mathbb{R}^{+}$. Suppose that $f(z) \in \mathcal{A}(p)(p \neq 1)$ satisfies the condition

$$
\left|\arg \left(\frac{f^{\prime}(z)}{p z^{p-1}}\right)\right|<\frac{\pi}{2}\left(\alpha+\frac{2}{\pi} \arctan \left(\frac{\alpha}{p-1}\right)\right),
$$

then

$$
\left|\arg \left(\frac{p-1}{p z^{p-1}} \int_{0}^{z} \frac{f^{\prime}(t)}{t} \mathrm{~d} t\right)\right|<\frac{\pi}{2} \alpha, \quad(z \in \mathbb{U}) .
$$

Finally, we consider the generalized Bernardi-Libera-Livingston integral operator $\mathcal{L}_{\sigma}(f)(\sigma>-p)$ defined by (cf. [8] [9] and [10])

$$
\mathcal{L}_{\sigma}(f) \equiv \mathcal{L}_{\sigma}(f)(z):=\frac{\sigma+p}{z^{\sigma}} \int_{0}^{z} t^{\sigma-1} f(t) \mathrm{d} t, \quad(f \in \mathcal{A}(p) ; \sigma>-p) .
$$

Theorem 3. Let $\lambda \geq 0, \mu<\min \{v+p+1, p\}, \sigma>-p$ and $\alpha, \gamma, \delta \in \mathbb{R}^{+}$, and let $g(z) \in \mathcal{A}(p)$. Suppose that $f(z) \in \mathcal{A}(p)$ satisfies the condition

$\left|\arg \left(\left\{\frac{\Delta_{z, p}^{\lambda, \mu, v} \mathcal{L}_{\sigma}(f)(z)}{\Delta_{z, p}^{\lambda, \mu, v} \mathcal{L}_{\sigma}(g)(z)}\right\}^{\gamma}\left\{1+\delta\left(\frac{\Delta_{z, p}^{\lambda+1, \mu+1, v+1} f(z)}{\Delta_{z, p}^{\lambda, \mu, v} \mathcal{L}_{\sigma}(f)(z)}-\frac{\Delta_{z, p}^{\lambda+1, \mu+1, v+1} g(z)}{\Delta_{z, p}^{\lambda, \mu, v} \mathcal{L}_{\sigma}(g)(z)}\right)\right\}\right)\right|<\frac{\pi}{2}\left(\alpha+\frac{2}{\pi} \arctan \left[\frac{\delta}{\gamma(\sigma+p)} \alpha\right]\right)$,

then

$$
\left|\arg \left\{\frac{\Delta_{z, p}^{\lambda, \mu, v} \mathcal{L}_{\sigma}(f)(z)}{\Delta_{z, p}^{\lambda, \mu, v} \mathcal{L}_{\sigma}(g)(z)}\right\}^{\gamma}\right|<\frac{\pi}{2} \alpha, \quad(z \in \mathbb{U}) .
$$

Proof. From (2.10) we observe that 


$$
z\left(\Delta_{z, p}^{\lambda, \mu, v} \mathcal{L}_{\sigma}(f)(z)\right)^{\prime}=(\sigma+p) \Delta_{z, p}^{\lambda, \mu, v} f(z)-\sigma \Delta_{z, p}^{\lambda, \mu, v} \mathcal{L}_{\sigma}(f)(z)
$$

If we let

$$
h(z)=\left\{\frac{\Delta_{z, p}^{\lambda, \mu v} \mathcal{L}_{\sigma}(f)(z)}{\Delta_{z, p}^{\lambda, \mu, v} \mathcal{L}_{\sigma}(g)(z)}\right\}^{\gamma}, \quad(\gamma \neq 0),
$$

then $h(z)=1+c_{1} z+c_{2} z^{2}+\cdots$ is analytic in $\mathbb{U}$, with $h(0)=1$ and $h^{\prime}(0) \neq 0$. Differentiating both sides of (2.14) logarithmically, it follows that

$$
\frac{1}{\gamma} \frac{z h^{\prime}(z)}{h(z)}=\frac{z\left(\Delta_{z, p}^{\lambda, \mu, v} \mathcal{L}_{\sigma}(f)(z)\right)^{\prime}}{\Delta_{z, p}^{\lambda, \mu, v} \mathcal{L}_{\sigma}(f)(z)}-\frac{z\left(\Delta_{z, p}^{\lambda, \mu, v} \mathcal{L}_{\sigma}(g)(z)\right)^{\prime}}{\Delta_{z, p}^{\lambda, \mu, v} \mathcal{L}_{\sigma}(g)(z)} .
$$

Hence, by applying the same arguments as in the proof of Theorem 1 with (2.13) and (2.15), we obtain

$$
|\operatorname{argh}(z)|<\frac{\pi}{2} \alpha, \quad(z \in \mathbb{U})
$$

which proves Theorem 3.

\section{Acknowledgements}

This work was supported by Daegu National University of Education Research Grant in 2014.

\section{References}

[1] Srivastava, H.M. and Buschman, R.G. (1992) Theory and Applications of Convolution Integral Equations. Kluwer Academic Publishers, Dordrecht, Boston and London.

[2] Samko, S.G., Kilbas, A.A. and Marichev, O.I. (1993) Fractional Integral and Derivatives, Theory and Applications. Gordon and Breach, New York, Philadelphia, London, Paris, Montreux, Toronto and Melbourne.

[3] Raina, R.K. and Srivastava, H.M. (1996) A Certain Subclass of Analytic Functions Associated with Operators of Fractional Calculus. Computers \& Mathematics with Applications, 32, 13-19. http://dx.doi.org/10.1016/0898-1221(96)00151-4

[4] Raina, R.K. and Choi, J.H. (2002) On a Subclass of Analytic and Multivalent Functions Associated with a Certain Fractional Calculus Operator. Indian Journal of Pure and Applied Mathematics, 33, 55-62.

[5] Srivastava, H.M. and Aouf, M.K. (1992) A Certain Fractional Derivative Operator and Its Applications to a New Class of Analytic and Multivalent Functions with Negative Coefficients. I and II. Journal of Mathematical Analysis and Applications, 171, 1-13.

[6] Lashin, A.Y. (2004) Applications of Nunokawa’s Theorem. Journal of Inequalities in Pure and Applied Mathematics, 5, 1-5. Art. 111.

[7] Goyal, S.P. and Goswami, P. (2010) Argument Estimate of Certain Multivalent Analytic Functions Defined by Integral Operators. Tamsui Oxford Journal of Mathematical Sciences, 25, 285-290.

[8] Bernardi, S.D. (1969) Convex and Starlike Univalent Functions. Transaction of the American Mathematical Society, 135, 429-446. http://dx.doi.org/10.1090/S0002-9947-1969-0232920-2

[9] Libera, R.J. (1965) Some Classes of Regular Univalent Functions. Proceedings of the American Mathematical Society, 16, 755-758. http://dx.doi.org/10.1090/S0002-9939-1965-0178131-2

[10] Srivastava, H.M. and Owa, S. (Eds.) (1992) Current Topics in Analytic Function Theory. World Scientific Publishing Company, Singapore, New Jersey, London, and Hong Kong. 
Scientific Research Publishing (SCIRP) is one of the largest Open Access journal publishers. It is currently publishing more than 200 open access, online, peer-reviewed journals covering a wide range of academic disciplines. SCIRP serves the worldwide academic communities and contributes to the progress and application of science with its publication.

Other selected journals from SCIRP are listed as below. Submit your manuscript to us via either submit@scirp.org or Online Submission Portal.
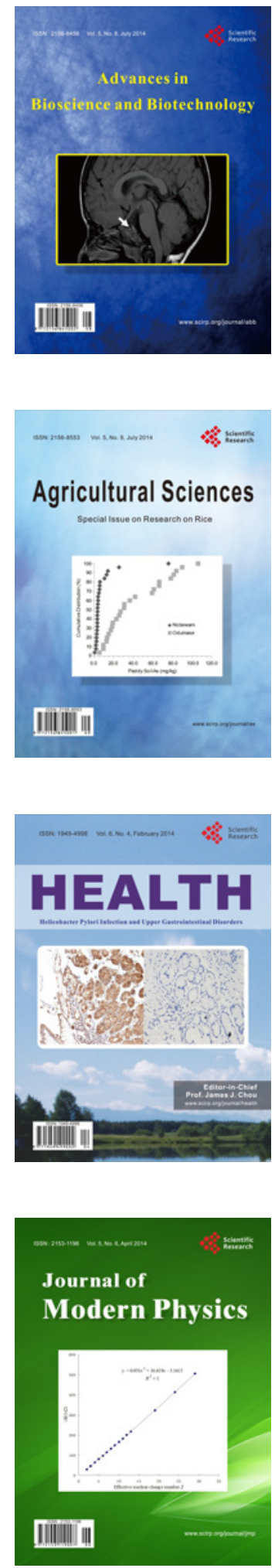
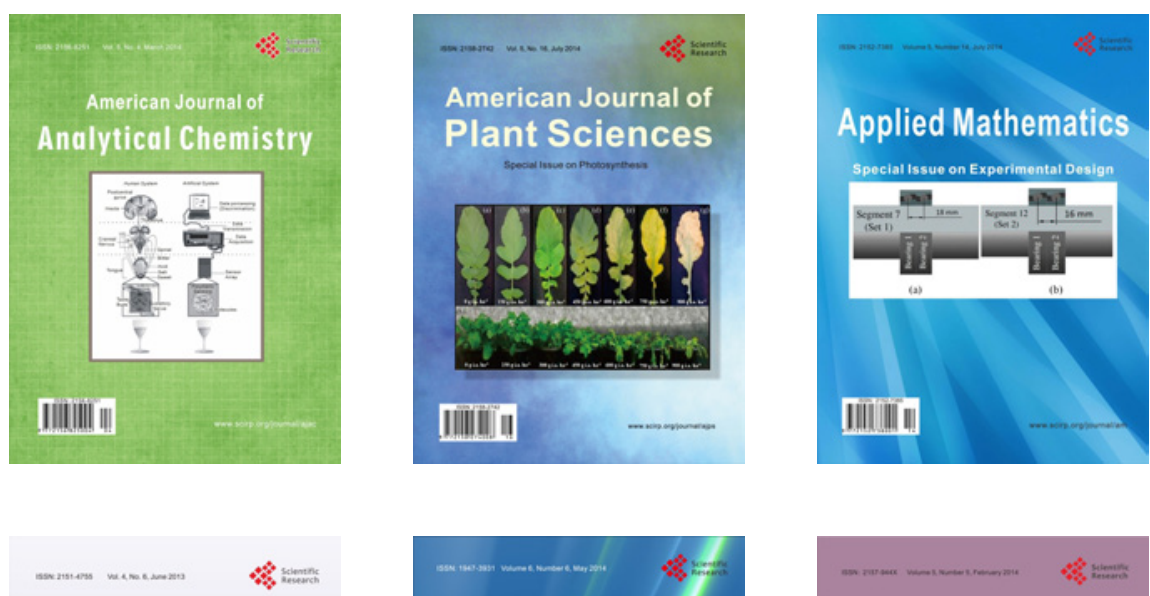

Creative Education
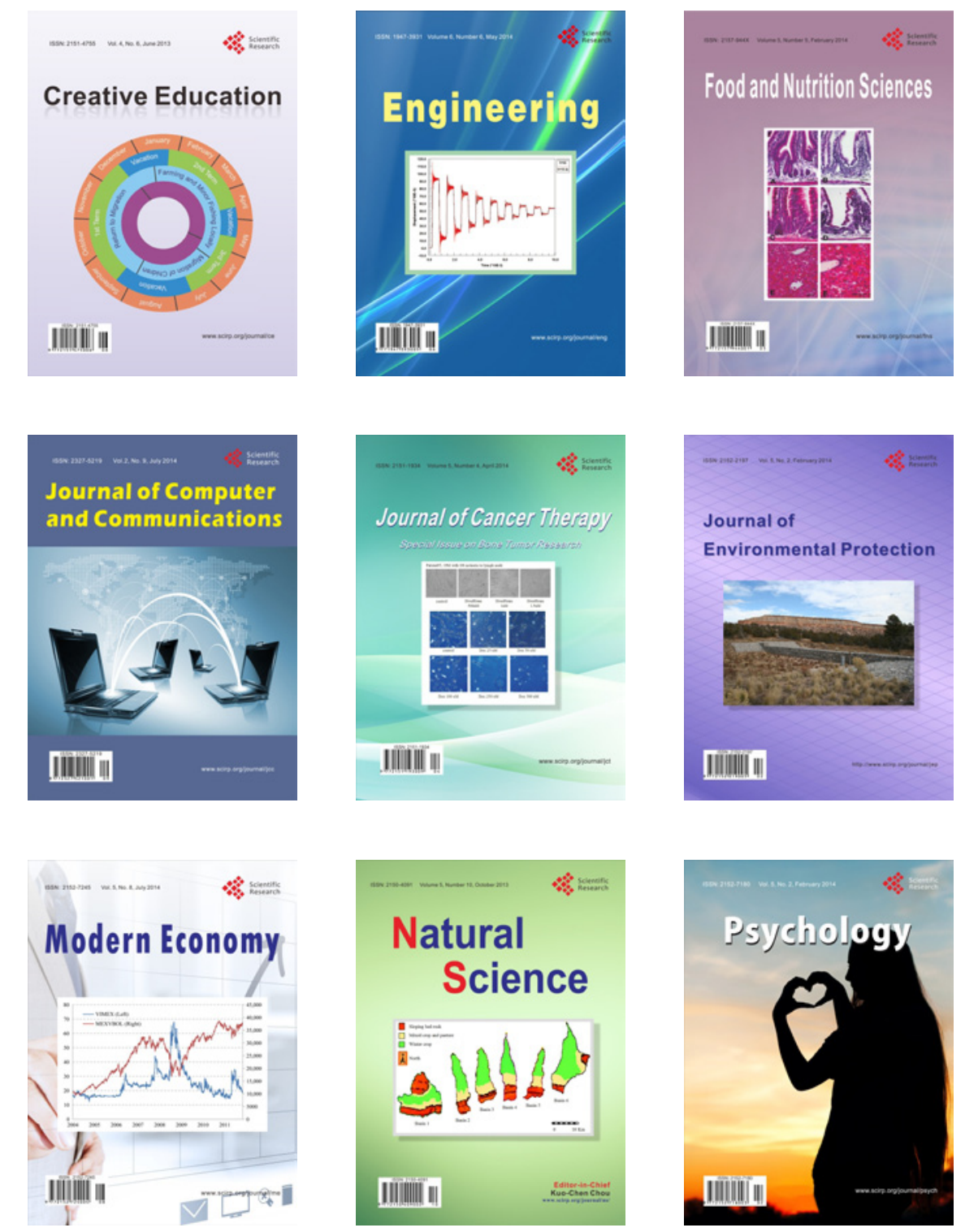